\title{
Development of a spherical stepping motor rotating around six axes
}

\author{
Akio Gofuku ${ }^{\mathrm{a}, *}$, Ryo Sasaki ${ }^{\mathrm{a}}$, Tomoaki Yano ${ }^{\mathrm{b}}$, Yosuke Wada ${ }^{\mathrm{a}}$ and Mitsunobu Shibata ${ }^{\mathrm{c}}$ \\ ${ }^{a}$ Graduate School of Natural Science and Technology, Okayama University, Okayama, Japan \\ ${ }^{\mathrm{b}}$ National Institute of Advanced Industrial Science and Technology, Japan \\ ${ }^{\mathrm{c}}$ Faculty of Engineering, Okayama University, Okayama, Japan
}

\begin{abstract}
Spherical motors have an advantageous feature that they can rotate around multiple axes. From the advantage, spherical motors are expected to be applied to driving mechanisms of holonomic mobile robots, joints of robots, and so on. This study develops a spherical motor called 14-12 spherical stepping motor that can rotate around 6 axes. This paper describes the structure of the motor, rotation principle by five-phase ACs (Alternative Currents), a five-phase AC power supply, and rotation experiments to confirm the applicability of the motor.
\end{abstract}

Keywords: Spherical motor, stepping motor, five-phase alternative current

\section{Introduction}

Spherical motors can rotate around multiple axes although usual motors rotate around only one axis. This characteristic feature of spherical motors gives several advantages compared with usual motors. A robot arm with three degrees of freedom needs at least three usual motors. The motor in the root side of the robot arm should move not only the load but also the other motors. This means that the energy efficiency of the total system should be low. On the other hand, because a spherical motor can move around multiple axes, a robot arm needs only one spherical motor in the root of the arm if only position control is considered. In addition, the rotation control of a spherical motor is easy compared with a combination system of several usual motors because the rotation center of a spherical motor coincides with its geometrical motor center. By considering the advantages of spherical motors, they are expected to be applied to driving mechanisms of holonomic mobile robots, joints of robots, stirrers for material production, incubators for biological cells, and so on.

Up to now, there are several types of spherical motors developed such as spherical supersonic motor [1], spherical induction motor [2], and spherical stepping motors [3-5]. However, there are deficiencies in each motor according to its working principle and structure. The surface of a spherical supersonic motor needs to be clean resulting in restricting its applications. The rotation angle error of the spherical induction motor depends on the rotation direction. The spherical stepping motors have small output rotation torques. To solve this problem, a group of spherical stepping motors that can have large output

\footnotetext{
${ }^{*}$ Corresponding author: Akio Gofuku, Graduate School of Natural Science and Technology, Okayama University, Japan 3-1-1, Tsushima-Naka, Kita-ku, Okayama, 700-8530, Japan. Tel.: +81 86251 8022; E-mail: fukuchan@ sys.okayama-u.ac.jp.
} 


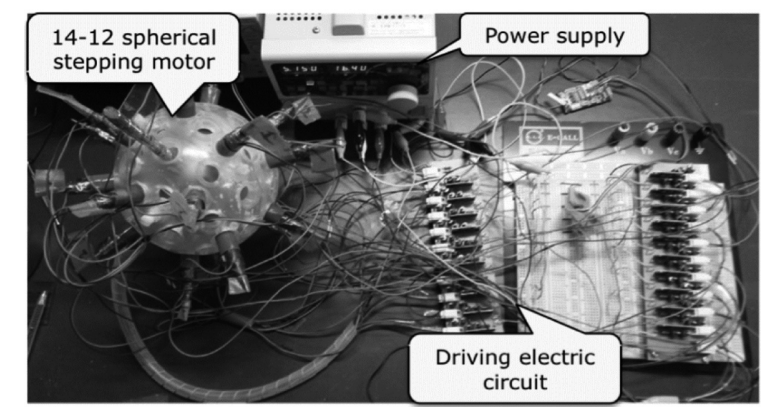

Fig. 1. Appearance of the total system of 14-12 spherical stepping motor. (Some electro-magnets are not assembled.)

rotation torque was proposed and a spherical stepping motor what is called 6-8 spherical stepping motor was developed [4]. In addition, the authors developed a spherical stepping motor [5] that rotates in any direction with some degrees of rotation angle errors and confirmed its applicability to stirrers [6].

This study develops a spherical stepping motor from the group having large output rotation torque and evaluates its basic rotation performance. The spherical stepping motor is called as 14-12 spherical stepping motor. This paper describes the structure of 14-12 spherical stepping motor, rotation principle by five-phase ACs (Alternative Currents), a five-phase AC power supply, and rotation experiments to evaluate basic rotation performance such as range of frequency of supplied five-phase AC for stable rotation, relation between rotation frequency and frequency of the supplied five-phase AC, and relation between rotation torque and frequency of the supplied five-phase $\mathrm{AC}$ for the rotation around one of two kinds of rotation axes.

\section{Structure of 14-12 spherical stepping motor}

\subsection{Total system}

The total system of 14-12 stepping motor is composed of a rotor, a stator, a driving electric circuit, and a commercial power supply as shown in Fig. 1. In the figure, some electro-magnets are not assembled. The 14-12 stepping motor can rotate around six axes. In the current version, the rewiring between the electro-magnets and the driving electric circuit are necessary when the axis and direction of rotation are changed. The rotation frequency is set beforehand in the program of micro-controller in the driving electric circuit. The control of the axis, direction, and frequency of rotation from PC is one of future problems. In the following subsections, the structure of the rotor and stator are described. Section 3 will give the explanation of the rotation principle by five-phase $\mathrm{AC}$ and the driving electric circuit.

\subsection{Rotor}

The rotor is a NAK80 spherical shell with 24 permanent magnets and 12 iron bolts covered by an acrylic spherical shell. Figure 2 shows the appearance of the rotor without the acrylic spherical shell. The outer and inner diameters of the NAK80 spherical shell are $62[\mathrm{~mm}]$ and $52[\mathrm{~mm}]$, respectively. On the other hand, the outer and inner diameters of the acrylic spherical shell are $78[\mathrm{~mm}]$ and $71[\mathrm{~mm}]$, respectively.

The cylindrical NdFeB permanent magnet that the diameter is $12[\mathrm{~mm}]$ and the length is $5[\mathrm{~mm}]$ is used. Its magnetic flux is $380[\mathrm{mT}]$. The permanent magnets are arranged at the vertices of a truncated regular 

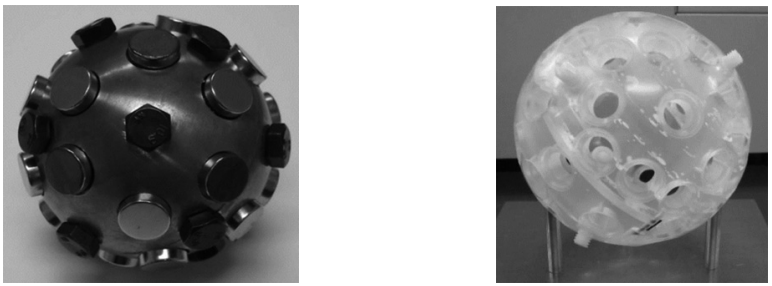

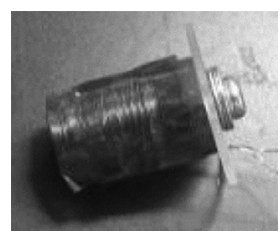

Fig. 2. Rotor without acrylic cover. Fig. 3. Stator without electro-magnets. Fig. 4. Electro-magnet. $\quad$ Fig. 5. Ball castor.

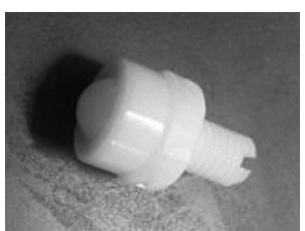

Fig. 2. Rotor without acrylic cover. Fig. 3. Stator without electro-magnets. Fig. 4. Electro-magnet. $\quad$ Fig. 5. Ball castor.

octahedron inscribed in the outer sphere of the NAK80 spherical shell. Twelve permanent magnets are placed such that their $\mathrm{N}$ poles direct outer the rotor. On the other hand, the remaining 12 permanent magnets are placed in reverse direction. An iron bolt is placed at the gravity center of each face, square or hexagon, for the purpose of fixing rotation axis by the attractive force of electro-magnets on the stator.

\subsection{Stator}

The stator is an acrylic shell with 62 electro-magnets to generate the rotation force, 6 ball castors to support the rotor, and 3 legs made by brass. The appearance of the stator without electro-magnets is shown in Fig. 3. The electro-magnets are placed at the vertices, the midpoints of neighboring two vertices, and the gravity centers of regular pentagon faces of the regular dodecahedron that is inscribed in the inner sphere of the acrylic spherical shell.

Each electro-magnet shown in Fig. 4 is made by winding a copper wire whose diameter is 0.2 [mm] for 1000 turns around an iron cylindrical bar whose diameter is $3[\mathrm{~mm}]$. The magnetic flux of the electro-magnet is 22.2 [mT] when the current of 0.1 [A] flows through the electro-magnet. The ball castors shown in Fig. 5 support the rotor with the gap of 4 [mm] from the rotor.

\section{Rotation principle}

\subsection{Rotation axis}

The 14-12 spherical stepping motor uses the solid geometry of truncated octahedron and regular dodecahedron for arranging permanent magnets and electro-magnets. Figure 6 shows these solid geometries. Though each face of dodecahedron is regular pentagon, truncated octahedron has two kinds of faces of regular hexagon and square. Therefore, there are two spatial relations when a face of dodecahedron is in parallel with a face of truncated octahedron as shown in Fig. 7.

When a regular pentagon of the dodecahedron is in parallel with a regular hexagon of the truncated octahedron, the spatial relation of the two faces is drawn as shown in Fig. 7(a). In this case, the regular dodecahedron and the truncated octahedron have a common rotation axis shown as axis 1 in Fig. 6. On the other hand, when a regular pentagon of the dodecahedron is in parallel with a square of the truncated octahedron as shown in Fig. 7(b). In this case, the regular dodecahedron and the truncated octahedron have another common rotation axis shown as axis 2 in Fig. 6. Both relations are the same of the spatial relation between the rotor and the stator of a planer stepping motor. From this consideration, the spherical stepping motor rotates by the same working principle as that of planer stepping motors as will be described in Sub-section 3.2.

Along the axis 2, there are four pairs of the same relations between the squares made by the vertices in the rotor and regular pentagons made by the vertices in the stator. Therefore, by the simplest consideration, 

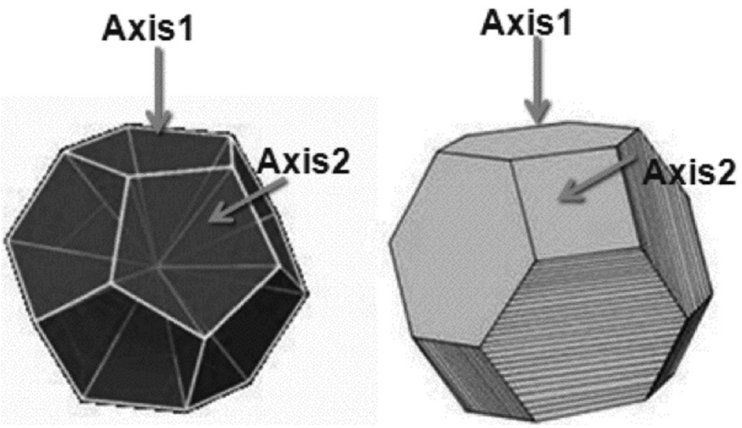

Fig. 6. Regular dodecahedron and truncated octahedron.

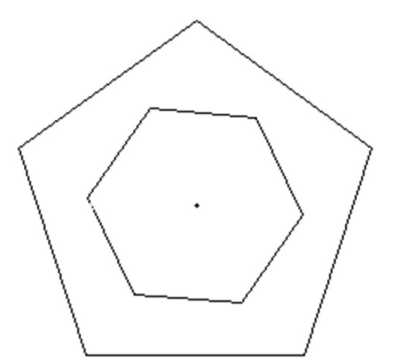

(a) Axis 1

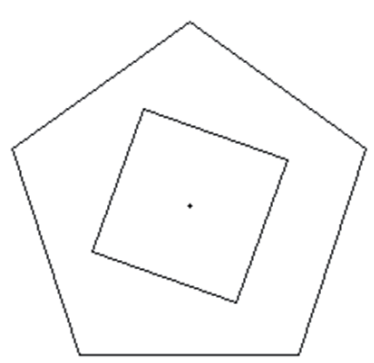

(b) Axis 2

Fig. 7. Relationship between two geometries.

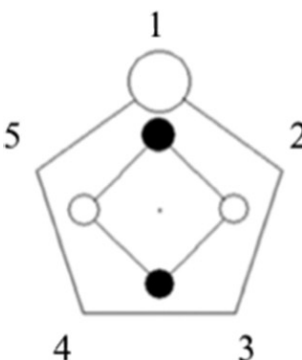

(1)

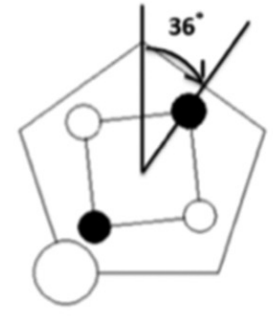

(2)

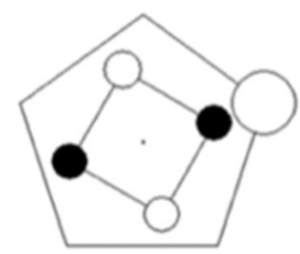

(3)

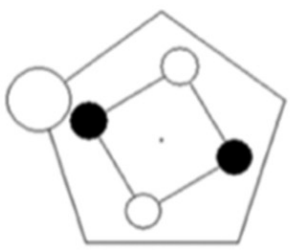

(4)

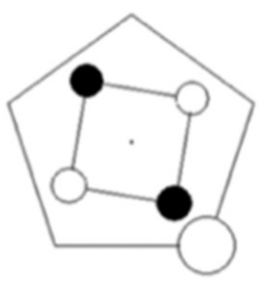

(5)

Fig. 8. Rotation of rotor by five-phase AC current.

the rotation torque will be four times as large as that of using only one of the pairs for rotation. In the same way, there are four pairs of the same relations along the axis 1 . Although the permanent magnets in the rotor form regular hexagons at the top and bottom along the axis 1, the permanent magnets in the middle of the rotor along the axis 1 form hexagons with two kinds of length of arcs. However, the rotation torque will be increased by suitably adjusting the excitation of electro-magnets.

\subsection{Rotation by five-phase $A C$}

The rotation principle of the 14-12 spherical stepping motor is briefly explained. The rotation around the axis 2 is first considered. In this case, a square face of the rotor is in parallel with the face of regular pentagon in the stator. The spatial relations among the vertices of the square face and those of the regular pentagon are drawn as shown in Fig. 8. In the figure, the black (white) small circles represent the permanent magnets that their $\mathrm{N}(\mathrm{S})$ poles are placed in the stator side.

When only the electro-magnet placed at the position 1 (electro-magnet 1) in the figure is excited to the $\mathrm{S}$ pole as shown in the condition 1 in Fig. 8, the rotor keeps the pose that one of permanent magnet whose $\mathrm{N}$ pole faces to the outside of the rotor stops at the nearest place to the electro-magnet 1 . Then, by exciting only the electro-magnet 4 to the $\mathrm{S}$ pole, the rotor rotates 36 [degrees] to the clockwise direction and stops in the pose shown as the condition 2 . In the same way, by exciting only the electro-magnet 2 , the rotor rotates to the clockwise direction by 36 [degrees]. In this way, the rotor rotates in the clockwise direction by exciting the electro-magnets in the order of 1, 4, 2, 5, and 3. From this working principle, the rotor continuously rotates by a five-phase AC. The rotation speed is determined by the frequency of the five-phase AC. When the five-phase AC is given for one cycle, the rotor rotates 180 [degrees]. Namely, the rotation frequency $f_{R 2}$ is the half of the frequency of the five-phase $\mathrm{AC}\left(f_{C}\right), f_{R 2}=1 / 2 \cdot f_{C}$. 


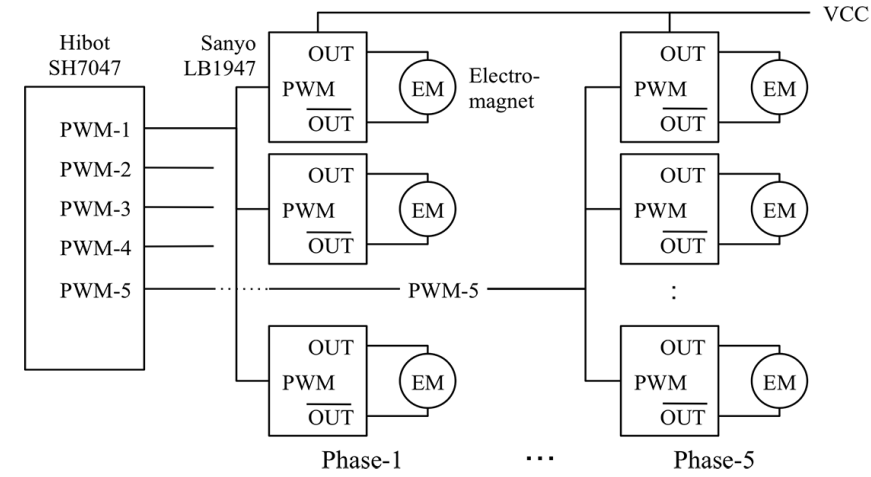

(a) Block diagram of the driving circuit

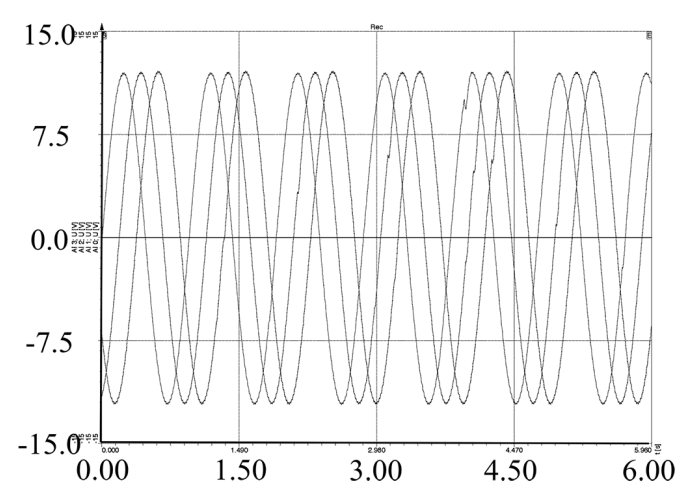

(b) Waveforms of output five-phase currents

Fig. 9. Block diagram of the driving circuit and waveforms of output five-phase currents.

From the same consideration, the rotor rotates 120 [degrees] around the axis 1 by giving a five-phase $\mathrm{AC}$ for one cycle. Therefore, the rotation frequency $f_{R 1}$ is given by $f_{R 1}=1 / 3 \cdot f_{C}$. However, the two hexagons made by the vertices in the middle of the rotor along the axis 1 are not regular hexagons but the hexagons that are composed of alternate two kinds of sides whose lengths are different. Therefore, if these hexagons are used for rotor rotation, it is necessary for a power supply to change the frequency of output $\mathrm{AC}$ between the former half and latter half of each cycle.

\subsection{Driving circuit of electro-magnets}

This study develops a driving circuit of electro-magnets that supplies five-phase AC. The circuit is composed of a micro-controller (Hibot SH7047), a DC power supply, and electric circuits for an electromagnet using a motor driver IC (Sanyo LB1947). The current version only supplies five-phase AC with a fix frequency set by the control program of the micro-controller. Figure 9 shows the block diagram of the driving circuit and the waveforms of output currents. Only the waveforms of three phases are shown due to the number of probes of the oscilloscope used.

\section{Basic evaluation of rotation performance}

\subsection{Rotation around axes}

The sequential pictures to show the rotation of the 14-12 stepping motor around the axis 2 are shown in Fig. 10. In this rotation experiment, the electro-magnets are only placed at the vertices of top and bottom pentagons perpendicular to the axis 2 . In addition, an output bar with rectangular thin celluloid plate is attached to the iron volt of the rotor to fix the rotation axis, to observe the rotation and to measure the rotation angle velocity.

\subsection{Rotation performance}

This study evaluates the following basic rotation performance: (1) range of frequency of supplied five-phase AC for stable rotation, (2) relation between rotation frequency and frequency of the supplied five-phase AC, and (3) relation between rotation torque and frequency of the supplied five-phase AC for 


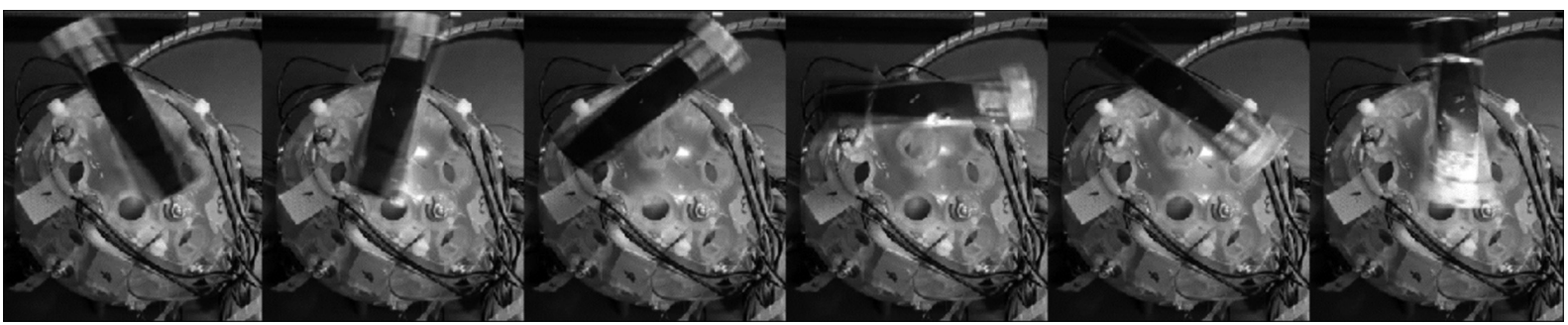

Fig. 10. Sequential pictures showing the rotation of 14-12 spherical stepping motor around the axis 2 .

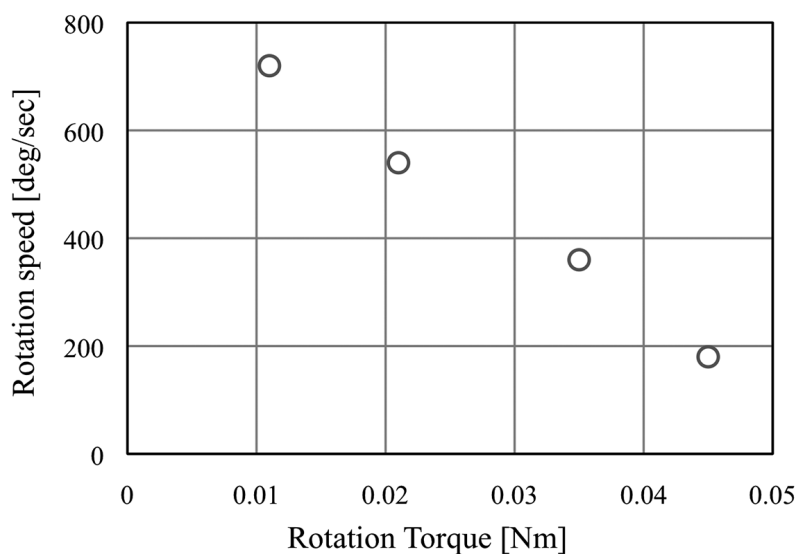

Fig. 11. Relationship between rotation torque and rotation speed for the rotation around axis 2 .

the rotation around the axis 2 . In the evaluations, the electro-magnets are installed at the vertices of four pentagons for the rotation of the axis 2 and the vertices of top and bottom pentagons for the rotation of the axis 1 .

The frequency ranges of five-phase AC that the motor rotates stably are 1 to $6[\mathrm{~Hz}]$ for the rotation around the axis 1 and 3 to $7[\mathrm{~Hz}]$ for the rotation around the axis 2 . The frequency range for the rotation around the axis 1 will increase if electro-magnets are arranged at the vertices of the second and third pentagons. The rotation frequencies are confirmed to coincide well with the theoretical values for the rotations around axes 1 and 2 . The measured rotation torques for several rotation speeds around the axis 2 are shown in Fig. 11. From the figure, the maximum rotation torque is 0.045 [Nm].

\section{Conclusions}

This study proposes a 14-12 spherical stepping motor as a high torque spherical motor and develops a proto-type motor system. Some evaluations of rotation performance exhibit the applicability of the 14-12 spherical stepping motor.

Future works include the improvement of the driving circuit of electro-magnets to supply a five-phase $\mathrm{AC}$ with changing its frequency in each cycle for some electro-magnets in rotating around the axis 1 , the development and implementation of the way to change rotation axis, and the evaluations of output rotation torque. 


\section{References}

[1] H. Kanazawa, T. Tsukimoto, T. Maeno and A. Miyake, Tribology of ultrasonic motor, J Japanese Society of Tribologists (in Japanese), 38(3) (1993), 207-212.

[2] B. Dehez, G. Galary, D. Greiner and B. Raucent, Development of a spherical induction motor with two degrees of freedom, IEEE Trans on Magnetics 42(8) (2006), 2077-2089.

[3] T. Yano and T. Suzuki, Basic characteristics of the small spherical stepping motor, Proc 2002 IEEE/RSJ Int Conf on Intelligent Robots and Systems (IROS'02) (2002), 1980-1985.

[4] T. Yano, Basic characteristics of a hexahedron-octahedron based spherical stepping motor, Proc Int Symp on Power Electronics, Electrical Drives, Automation and Motion (Speedam2010) (2010), 748-753.

[5] S. Ikeshita, A. Gofuku, T. Kamegawa and T. Nagai, Development of a spherical motor driven by electro-magnets, $J$ of Mechanical Science and Technology 24(1) (2010), 43-46.

[6] W. Shan, A. Gofuku, M. Shibata, T. Yano and T. Kamegawa, A stirrer driven by a spherical stepping motor, Proc ISEF 2011 - XV Int Symp on Electromagnetic Fields in Mechatronics, Electrical and Electronic Engineering, 2011. 